\title{
Testicular Dose During Prophylaxis of Heterotopic Ossification with Radiation Therapy
}

\author{
WALEED F. MOURAD ${ }^{1,2}$, JOHN K. MA ${ }^{1}$, SATYASEELAN PACKIANATHAN ${ }^{1}$, WEISI YAN ${ }^{3}$, \\ SHERIF G. SHAABAN ${ }^{2}$, EDWARD M. MARCHAN ${ }^{2}$, LAMIAA E. ABDALLAH ${ }^{2}$, \\ REI HE ${ }^{1}$, PAUL N. MOBIT ${ }^{1}$, CHUNLI (CLAUS) YANG ${ }^{1}$ and SRINIVASAN VIJAYAKUMAR ${ }^{1}$ \\ ${ }^{1}$ Department of Radiation Oncology, University of Mississippi Medical Center, Jackson, MS, U.S.A.; \\ ${ }^{2}$ Department of Radiation Oncology, Georgia Cancer Center, \\ Medical College of Georgia, Augusta University, Augusta, GA, U.S.A.; \\ ${ }^{3}$ Department of Radiation Oncology, University of South Alabama, Mobile, AL, U.S.A.
}

\begin{abstract}
Aim: A single-institution, retrospective study was performed to investigate potential techniques to minimize radiation exposure to the testicles during heterotopic ossification $(\mathrm{HO})$ prophylaxis. We report the impact of splitbeam technique (SBT) and different photon energies on the total dose of radiation received by the testicles during prophylaxis of HO. Materials and Methods: Between 2008 and 2010, we identified 64 patients with traumatic acetabular fractures who underwent surgery followed by radiation therapy $(R T)$ without testicular shielding. Postoperative RT was delivered within $72 \mathrm{~h}$ in a single fraction of $700 \mathrm{cGy}$ using 6-18 MV photons, without testicular shielding due to patient refusal. All patients underwent 3-D RT planning in which the testicles were contoured as a region of interest and dosevolume histograms (DVH) were generated. Additional treatment planning trials were created for each patient by utilizing a SBT medially and by using different photon energies (6, 10 and $18 \mathrm{MV}$ ) to study the effects of these maneuvers on the delivered dose to the testicles. Results: In reviewing the $D V H$, it was noted that the mean dose delivered to the testicles was 10 cGy (range=3-40). The maximum dose was 31 cGy (range=7-430). When SBT was utilized, a
\end{abstract}

This article is freely accessible online.

Presented at the American Society for Therapeutic Radiology and Oncology (ASTRO) 56th Annual Meeting 2014.

Correspondence to: Waleed F. Mourad, MD, Department of Radiation Oncology, Georgia Cancer Center, Medical College of Georgia, Augusta University, Augusta, GA, USA. Tel: +1 3477827886, e-mail: Waleed246@gmail.com

Key Words: Radiation therapy (RT), acetabular fracture, heterotopic ossification (HO), megavoltage photon, split-beam technique, testicular dose, radiation prophylaxis, indomethacin. significant reduction in the mean (44\%) and maximum (47\%) doses delivered to the testicles was noted. Further reductions in the mean (26\%) and maximum (14\%) doses were achieved by using higher-energy (10-18 MV) beams. The radiation doses to the testicles from the CT simulation and the two portal images were estimated to be 4 and 1.5 cGy, respectively. Conclusion: Low-dose prophylactic $R T$ to prevent $H O$ around the hip causes a low, but likely biologically meaningful, radiation dose to be delivered to the testicles. This dose could be further reduced by using a medial SBT and photon energies above $6 \mathrm{MV}$. Testicular shielding should be offered to all male patients receiving such RT. In addition, all patients should be informed about the consequences of testicular radiation as part of their informed consent.

Heterotopic ossification ( $\mathrm{HO}$ ) is the pathological process of bone formation in non-osseous tissues following trauma and/or surgical intervention. Following traumatic injury, cellular and hormonal signals from the site of injury, a supply of mesenchymal cells and the appropriate tissue microenvironment are all among the potential factors that could contribute to development of HO in injured tissues (1). Prophylactic radiation therapy (RT) is commonly used to reduce the risk of $\mathrm{HO}$ after open reduction and internal fixation (ORIF) of traumatic acetabular fractures (TAFs); heterotopic ossification after operative treatment of the hip using a posterior surgical approach has been reported in up to $50 \%$ of such patients (2). Radiation therapy presumably decreases the risk of $\mathrm{HO}$ by inhibiting the proliferation of pluripotential mesenchymal cells that could potentially differentiate into osteoblastic stem cells and is usually administered postoperatively, within $72 \mathrm{~h}$ of surgery (3-5). However, preoperative RT has also been used in these situations (6-8). In a recent meta-analysis reporting the incidence of $\mathrm{HO}$ after TAF status post ORIF, it was reported that the incidence of $\mathrm{HO}$ formation after RT prophylaxis 
alone was $27 \%, 37 \%$ after indomethacin prophylaxis alone and $12 \%$ after both RT combined with indomethacin. The incidence of $\mathrm{HO}$ was $58 \%$ in the control arm (i.e., patients who did not receive any form of prophylaxis) $(4,9)$. Most patients who develop $\mathrm{HO}$ are males with a TAF. The testes, given their proximity to the pelvis, are always exposed to scatter radiation from $\mathrm{RT}$, which has the potential to alter sperm count and morphology. Since sperm abnormalities have been reported with doses of only $15 \mathrm{cGy}$, all males should be counseled and treated with a testicular shield (10, $11)$ in place $(12,13)$. Here we report our testicular dose estimates and discuss techniques that can optimize coverage while delivering the smallest testicular dose.

\section{Materials and Methods}

Study objective and schema. For this analysis, we conducted a retrospective study to assess the RT doses to the scrotum/testicles during single-fraction treatment for $\mathrm{HO}$ prevention and investigated techniques that could reduce the dose to the scrotum/testes in patients who decline, for whatever reason, to allow the use of a testicular shield. We hypothesized that the scattered dose to the scrotum/testicles would be minimized by utilizing a split-beam technique (SBT) medially and, by using higher photon energies (10 and $18 \mathrm{MV}$ ), we could consequently diminish most RT-induced scattered dose.

Patient population. This study represents a single-institution; retrospective investigation performed at the University of Mississippi Medical Center, Jackson, MS, USA, and was fully approved by our Institutional Review Board.

Patient eligibility and design. Between 2008 and 2010, 64 male patients who had been treated in standard operative fashion for TAF at the Department of Orthopedic Surgery were then referred to the Department of Radiation Oncology for prophylaxis of HO with RT. All patients had experienced a TAF that required ORIF. RT prophylaxis was delivered with or without indomethacin. For all patients, the following data were tabulated (Table I): age, types and causes of fracture, surgical approach used, RT and usage of indomethacin.

Surgical treatment. All surgical procedures were performed by fellowship trained, orthopedic trauma surgeons. All patients underwent ORIF via a posteriorly approached (Kocher-Langenbeck) surgical exposure with the patient in either lateral or prone position $(15,16)$. Early postoperative patient mobilization, with toe-touch weight bearing as tolerated, was initiated for all patients.

Radiation treatment. RT was used prophylactically for all the patients and delivered within $72 \mathrm{~h}$ after the ORIF. Seven hundred cGy was delivered in a single fraction to the mid-plane, using 6-18 MV photons. Treatment fields included the soft tissues around the proximal femur and acetabulum without bone shielding (15-17). Five different treatment plans were generated after CT simulation (the process used to determine the exact location, shape and size of the targeted area to be treated) to investigate the testicular doses. The first plan was generated by placing the isocenter in the middle of the
Table I. Patient characteristics.

\begin{tabular}{lc}
\hline Patients & Total \# 64 \\
\hline MVC & $100 \%$ \\
Male & $100 \%$ \\
Age & $33(16-65)$ \\
BMI & $28(20-46)$ \\
Left hip $v s$. right hip & $48 \%$ vs.52\% \\
Separation & $23(17-34)$ \\
Mean (Mean dose) cGy & $10(3-40)$ \\
Mean (Maximum dose) cGy & $31(7-430)$ \\
Field size X x Y in cc & $4,634(2,240-8,488)$ \\
Fracture - Surgery in days & $2(0-20)$ \\
Fracture - RT in days & $4(2-21)$ \\
Surgery - RT in days & $2(1-4)$ \\
Indomethacin & $40 \%(25 / 64)$ \\
CT-based 3-DRT & $100 \%$ \\
700 cGy & $100 \%$ \\
Posterior wall fracture & $69 \%(44 / 64)$ \\
Posterior wall and T-shaped fracture & $22 \%(15 / 64)$ \\
T-shaped fracture & $9 \%(5 / 64)$ \\
Kocher-Langenbeck approach (14, 15) & $100 \%$ \\
HO & $5 \%(3 / 64)$ \\
Brooker (17) II and III & $67 \%(2 / 3)$ and 33\% (1/3) \\
\hline
\end{tabular}

MVC, Motor vehicle crash; CT, computerized tomography; cGy, centiGray; 3-DRT, three-dimensional radiation therapy; BMI, body mass index; $\mathrm{HO}$, heterotopic ossification.

field, the second plan by applying a SBT (e.g. half-beam block) wherein the isocenter was placed at the fractured acetabulum (Figure 1). Subsequently, three different plans were generated for each isocenter using 6,10 and $18 \mathrm{MV}$ photons for comparison. Dosevolume histograms (DVHs) were generated for evaluation of the computed scattered dose delivered to the testicles (Figure 2).

Medical treatment. Forty percent (25/64 patients) of the whole cohort received RT with indomethacin. Indomethacin was prescribed at the discretion of the surgeon at the dosage of $25 \mathrm{mg}$ three times per day after meals, beginning on postoperative Day 1 and continued for 6 weeks thereafter; however, patient compliance with daily intake was not verified.

Follow-up. Patients had follow-up appointments at 2 weeks, 1 month, 3 months, 6 months and 1 year from the day of discharge. Standard hip X-rays (AP, PA and oblique) were obtained for evaluation of HO per the Brooker classification (18), avascular necrosis of the femoral head or loosening, malunion or non-union of the fracture. Computed tomography of the pelvis was obtained where considered clinically appropriate. All patient medical records and X-rays were reviewed to determine the efficacy of the RT in preventing HO.

Hypothesis and study end-points. In this study, we hypothesized that by utilizing a SBT medially and using higher photon energies $(10$ and $18 \mathrm{MV}$ ) we could minimize the scattered dose to the scrotum/testicles and, thereby, minimize RT-induced testicular damage. 


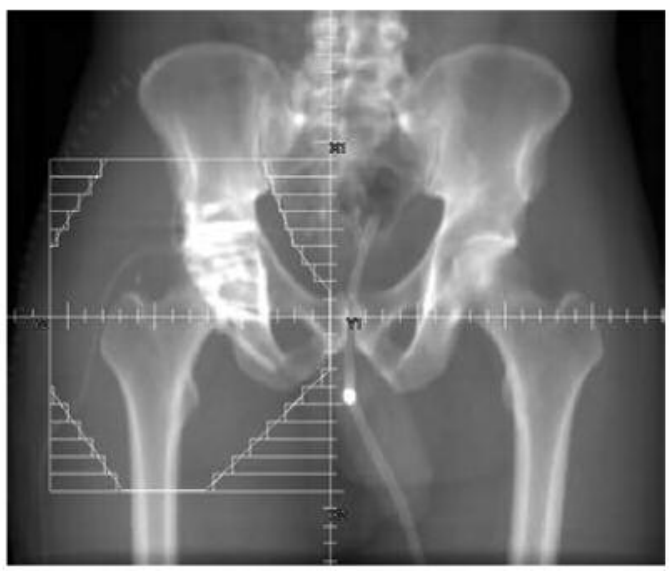

Figure 1. Split-beam technique (SBT, e.g. half-beam block.

Table II. The impact of beam-split technique (BST) and different photon energies $(6,10,18 \mathrm{MV})$ on testicular dose.

\begin{tabular}{lcc}
\hline Energy (cGy) & $\begin{array}{c}\text { Mean (Mean dose) } \\
10(3-40)\end{array}$ & $\begin{array}{c}\text { Mean (Maximum dose) } \\
31(7-430)\end{array}$ \\
\hline $6 \mathrm{X}$ & 13.5 & 37.9 \\
$10 \mathrm{X}$ & 10.6 & 35.5 \\
$18 \mathrm{X}$ & 8.3 & 29.9 \\
\hline
\end{tabular}

Dose reduction- SBT $(p<0.001)$

\begin{tabular}{lcc}
\hline Energy & Mean (Mean dose) & Mean (Maximum dose) \\
\hline $6 \mathrm{X}, 10 \mathrm{X}, 18 \mathrm{X}$ & $44 \%$ & $47 \%$ \\
\hline
\end{tabular}

Dose reduction - Energy $(p<0.001)$

\begin{tabular}{lcc}
\hline Energy & Mean (Mean dose) & Mean (Maximum dose) \\
\hline $6 \mathrm{X} v$ s. 10X & $22 \%$ & $6.5 \%$ \\
$6 \mathrm{X} v$ s. 18X & $39 \%$ & $21 \%$ \\
$10 \mathrm{X} v$ s. $18 \mathrm{X}$ & $22 \%$ & $15 \%$ \\
\hline
\end{tabular}

Statistical methods. Univariate and multivariate regression analyses were used to compare testicular doses received in the different groups, after adjusting for all other factors that could potentially affect HO formation (Body mass index (BMI), tissue separation, type of fracture and surgical approach to fracture exposure).

\section{Results}

The median patient age was 33 years (range=16-65). Motor vehicle crashes (MVC) (100\%) was the cause of the fractures in all our patients. The median BMI was 28 (range=20-46). Posterior acetabular wall and combined posterior wall and T-

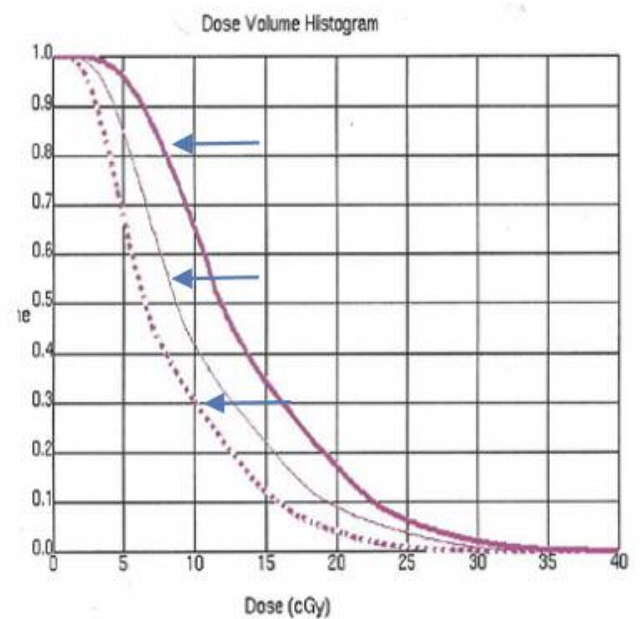

\begin{tabular}{|ccc|} 
& Mean Dose cGy & Maximum Dose cGy \\
\hline 6X -Thick Line & 13.5 & 37.9 \\
10X- Thin line & 10.6 & 35.5 \\
18X - Dashes & 8.3 & 29.9
\end{tabular}

Figure 2. Dose-volume histogram shows the impact of beam-split technique (BST) and different photon energies (6-thick line, 10-thin line, 18-dashes MV) on testicular dose.

shaped fractures were the types of injury seen in the majority of patients. All patients were males and all underwent ORIF via the Kocher-Langenbeck approach; thereafter, all underwent CT-based simulation and 3-D RT planning. At a median follow-up of 3 years for the whole cohort, the incidence of $\mathrm{HO}$ in all patients was only 5\% (3/64) with RT with/without indomethacin.

The univariate regression test was applied first to test for an association between testicular dose reduction and SBT and different photon energies before adjusting for other factors. There was a significant reduction in the testicular dose $(p<0.0001)$ with SBT. Further, multivariate regression analysis confirmed the significant reduction in testicular dose after adjusting for all other factors (age, BMI, separation, size of the RT field, types of fracture and surgical exposures) that could potentially impact the testicular dose.

In reviewing the DVH for the 64 patients (Figure 2, Table II), it was noted that the mean (Mean dose) delivered to the testicles using the three different beam energies was $10 \mathrm{cGy}$ (range=3-40), while the mean (Maximum dose) from the three beam energies was $31 \mathrm{cGy}$ (range=7-430). When a SBT was applied at the medial edge of the field, a significant reduction in the mean (Mean dose) (44\%) and mean (Maximum dose) (47\%) dose delivered to the testicles was noted. Moreover, additional dose reductions in the mean 
(Mean dose) of $26 \%$ and mean (Maximum dose) of $14 \%$ were achieved by using a higher beam energy (10 or 18 $\mathrm{MV}$ ), when compared to $6 \mathrm{MV}$ photons. The radiation doses to the testicles from the CT simulation and the two portal images were estimated at 4 and $1.5 \mathrm{cGy}$, respectively.

\section{Discussion}

$\mathrm{HO}$ is the pathological process of bone formation in nonosseous tissues following trauma and/or surgical intervention. This misplaced growth occurs between muscle planes and not within the muscle fibers themselves (18). HO was first identified and reported in the literature in 1883 by Riedel (20), a German physician. It was later observed by the French physicians Dejerne and Ceillier (21) in patients suffering traumatic paraplegia in World War I. There are several factors that appear critical to HO formation - a traumatic injury, signals from the site of injury, a supply of mesenchymal cells and the appropriate tissue microenvironment - all appear to be factors that may contribute to the development of $\mathrm{HO}$ in injured tissues (1). The most common procedures that result in $\mathrm{HO}$ are: ORIF of an acetabular fracture or a total hip arthroplasty (THA) $(4,9)$. Many clinicians recommend prophylactic RT and/or non-steroidal anti-inflammatory medications (NSAIDs) to prevent HO.

Radiation therapy presumably decreases the risk of $\mathrm{HO}$ by inhibiting the proliferation of pluripotential mesenchymal cells that could potentially differentiate into osteoblastic stem cells and is usually given postoperatively, within 72 hours of surgery or preoperatively (3-8). We hypothesized that by utilizing a SBT medially and/or by using higher photon energies (10 and $18 \mathrm{MV}$ ), we could minimize the scattered dose to the scrotum/testicles and, consequently, diminish the RT-induced testicular damage. In order to achieve minimal dose divergence at the beam edge, a split beam technique, sometimes called a half-beam block, is often used. In this method, the beam is split along the plane containing the central axis inhibiting geometric divergence of the beams at the split line (22). This technique is often employed in matching one field to another. By applying a half-beam block in the medial edge of the pelvic AP/PA fields in $\mathrm{HO}$ prophylaxis, much of the dose that can potentially diverge into the testes, bowel and bladder is diverted laterally.

Most patients who develop HO are males after a TAF. The testes, given their proximity to the pelvis, will be exposed to scattered radiation, which has the potential to alter sperm count and morphology. Radiobiological studies have shown that the testicles are very radiosensitive and very low doses of radiation have been shown to alter sperm production and morphology and cause transient or even total azoospermia/sterilization (11). The largest study (53 patients) evaluating testicular dose and sperm function was conducted by the Southwest Oncology Group (27). The median gonadal dose in this study was 79 cGy. Recovery of fertility occurred approximately 1 year after radiation, which was both doseand time-dependent. Hall et al. (11) have reported a temporary reduction in the number of spermatozoa with radiation doses as low as $10 \mathrm{cGy}$ and temporary sterility at doses of 15 cGy. Moreover, Freund et al. (29) reported on testicular function dysfunction in 8 seminoma patients treated with radiation, where the absorbed gonadal radiation dose ranged from 15 to $157.5 \mathrm{cGy}$.

In a prospective study by Patel et al., patients were treated with 800 cGy without direct exposure of the testes/scrotum and while using a testicular shield. Thermoluminescent dosimeters were placed inside and outside the shield. The mean dose inside and outside the shield was 10.2 and 20.2 cGy, respectively. The authors concluded that young males should be counseled of the risks of testicular radiation and treated with a testicular shield (10). Bieri et al. have reported that the daily dose to the testes was $1.86 \mathrm{cGy}$ per fraction without gonadal shielding and $0.65 \mathrm{cGy}$ per fraction with gonadal shielding for males with seminoma treated at 1.8 Gy per fraction to a para-aortic field, suggesting that a scrotal shield should be used to reduce the scatter dose to help avoid impairment of spermatogenesis (27). For male patients presenting for RT prophylaxis of $\mathrm{HO}$, it is standard practice to routinely offer testicular shielding for all patients who want to preserve their fertility or the ability to conceive, since the RT field is in close anatomical proximity to the testes $(12,13)$.

In a study utilizing dose modeling in phantoms, the estimated total gonadal dose and risk of hereditary effects during therapeutic external irradiation was shown to be $\sim 39$ cGy using $6 \mathrm{MV}$ photons without the SBT technique; this was associated with a risk for genetic effects in offspring of $23.4 \times 10^{-4}$. This is 4 times greater than the risk generated from a typical computed tomography scan of the abdomen, which was estimated to be $6 \times 10^{-4}$ in males (14). Generally, most radiation oncologists would agree that side-effects, such as temporary azospermeia and sperm chromosomal abnormalities from scattered dose to the testes, could happen with very low RT doses in the range of 10 to 15 cGy (10). Thus, using a SBT with higher photon energy, as we have reported, has the potential to reduce risk for genetic effects in offspring by two thirds.

Some male patients specifically decline to use the clam shell testicular shield, as did all the patients in this study. Others may be so obese that the standard shell will not fit comfortably between the excess tissues in the thighs of the patient. For such patients then, we must use non-shield techniques to obtain adequate coverage of the targeted area while providing the least integral dose to the testicles. In the current study, we retrospectively evaluated how one could minimize the RT doses to the scrotum/testicles during singlefraction treatment for $\mathrm{HO}$ prevention. 
We were able to minimize the testicular dose by applying SBT and higher photon energies. There was a significant reduction $(>50 \%)$ of testicular dose by utilizing these simple-touse maneuvers. Using SBT and $\geq 10 \mathrm{MV}$ photon beams led to a smaller scattered RT dose compared to lower energy $6 \mathrm{MV}$ photons. Higher energies lead to a more consistently forward directed beam and decreased side scattering. The major caveat of using high energy $18 \mathrm{MV}$ photons, however, is the neutron contamination that occurs when beams with $>10 \mathrm{MV}$ energy are used. The dose contribution to the testicles from neutron contamination in $15 \mathrm{MV}$ or $18 \mathrm{MV}$ photons is difficult to quantify. For this reason, beams of energy $15 \mathrm{MV}$ and higher should also be avoided due to the increased neutron contamination from beam modifying components of high atomic number, such as the target and jaws (30). In conjunction with a "half-beam blocking" technique, one can also employ a physical shield to protect the testes from scattered radiation during treatment as has been demonstrated by Fraass and colleagues (30). They described the use of testicular shielding for testicular dose reduction in 1985. Shields are commercially available and, while considered to be a standard part of therapy when treating young males in the setting of Hodgkin's disease or seminoma, they have not been used as commonly, during $\mathrm{HO}$ prophylaxis.

Mourad et al., in 2010, presented the first radiationinduced sarcoma case in the literature after RT prophylaxis of $\mathrm{HO}$. The possibility of second malignancy after RT, especially in younger patients, is an additional concern and should be discussed with the patients. However, it is a rare side-effect and only 2 cases of radiation-induced sarcoma have been reported in the literature to date (23-26). There are two basic approaches to reduce the RT scatter dose effect on the testes. One approach is to improve the treatment delivery so as to decrease the amount of scattering dose, which can be achieved by using CT simulation, SBT and photon energies greater than $6 \mathrm{MV}(27,22)$. This effect is mainly a result of the decrease in penumbra and lateral scattering when going from lower to higher energy beams.

In summary, our results suggest that the use of field modification and higher beam energies during RT for HO prophylaxis appears to consistently reduce the radiation dose to the scrotum/testicles by about two- to three-fold of the values obtained using standard blocks and lower beam energy. This could translate into a by two- to three-fold reduction of risk for genetic effects in offspring. We also advocate the routine use of a testicular shield for all male patients receiving radiation for $\mathrm{HO}$ prophylaxis. Although, a testicular shield is effective in shielding scatter from external (machine head) sources, it does little to prevent in-scatter into the testicular region from within the patient. Hence, in order to further minimize the gonadal dose while maximizing sufficient target volume coverage, we recommend that CTbased simulation, 3-D treatment planning, a SBT and $\geq 10$ MV photon beams be utilized $(28,32-34)$.

\section{Conclusion}

Although a biologically meaningful radiation dose appears to be delivered to the testicles during low-dose prophylactic RT to prevent HO around the hip, this dose could be further reduced by minimizing the side scattering via the use of a medial SBT and photon energies $\geq 10 \mathrm{MV}$. Moreover, testicular shielding should be offered to all young male patients receiving $\mathrm{HO}$ prophylaxis using RT. Informed consent of the patient should include possible alteration in sperm production and morphology. Use of CT simulation and 3-D treatment planning will allow more accurate delineation of the tissues to be irradiated and, consequently, improved precision in RT delivery.

\section{Conflicts of Interest}

All Authors do not have any conflict of interest. There is no copyrighted information.

\section{References}

1 Kaplan FS, Glaser DL, Hebela N and Shore EM: Heterotopic ossification. J Am Acad Orthop Surg 12: 116-125, 2004.

2 Haas ML, Kennedy AS, Copelan CC, Ames JW, scarboro M and Slawson RG: Utility of radiation in the prevention of heterotopic ossification following repair of traumatic acetabular fracture. Int J Radiat Oncol Biol Phys 45: 4614-66, 1999.

3 Childs HA, Cole T, Falkenberg E, Smith JT, Alonso JE, Stannard JP, Spencer SA and Fiveash J: A prospective evaluation of the timing of radiotherapy for preventing heterotopic ossification following traumatic acetabular fractures. Int J Radiat Oncol Biol Phys 5: 1347-1352, 2005.

4 Pakos E and Ioannidis J: Radiotherapy vs. nonsteroidal antiinflammatory drugs for the prevention of heterotopic ossification after major hip procedures: A meta-analysis of randomized trials. Int J Radiat Oncol Biol Phys 3: 888-895, 2004.

5 Balboni TA, Gobezie R and Mamon HJ: Heterotopic ossification: Pathophysiology, clinical features, and the role of radiotherapy for prophylaxis. Int J Radiat Oncol Biol Phys 65: 1289-1299, 2006.

6 Lonardi F, Gioga G, Coeli M, Ruffo P, Agus G, Pizzoli A and Campostrini F: Preoperative, single-fraction irradiation for prophylaxis of heterotopic ossification after total hip arthroplasty. Int Orthop 25: 371-374, 2001.

7 Seegenschmiedt $\mathrm{M}$, Makoshki $\mathrm{H}$ and Micke O: Radiation prophylaxis for heterotopic ossification about the hip joint A multicenter study. Int J Radiat Oncol Biol Phys 51: 756-765, 2001.

8 Rumi MN, Deol GS, Bergandi JA, Singapuri KP and Pellegrini VD: Optimal timing of preoperative radiation for prophylaxis against heterotopic ossification: A rabbit hip model. Bone Joint Surg Am 87: 366-373, 2005.

9 Baird EO and Kang QK: Prophylaxis of heterotopic ossification - Review. J Orthopaedic Surg Res 4: 12, 2009.

10 Patel H, Silverman CL, Carrascosa L, Malkani A and Yashar CM: Evaluation of scrotal and testicular radiation doses for heterotopic ossification prophylaxis. Am J Orthop 37(9): E163-E166, 2008. 
11 Hall EJ: Radiobiology for the Radiologist 7th ed. Boston, MA: Lippincott Williams \& Wilkins, 2012.

12 Halperin EC, Perez CA and Brady LW: Principles and practice of radiation oncology 5th ed. Philadelphia, PA: Lippincott Williams \& Wilkin, 2008.

13 Gunderson LL and Tepper JE: Clinical radiation oncology: Expert Consult 3rd edition. Elsevier Saunders, 2012.

14 Kokona G1, Mazonakis M, Varveris H, Liraraki E and Damilakis $\mathrm{J}$ : Treatment of benign diseases with megavoltage $\mathrm{X}$-ray beams: Is there a risk for gonadal damage? Clin Oncol (R Coll Radiol) 18(9): 658-662, 2006.

15 Mourad WF, Packianathan S, Shourbaji RA, Zhang Z, Graves M, Khan MA, Baird MC, Russell G and Vijayakumar S: The impact of BMI on heterotopic ossification. Int J Radiation Oncol Biol Phys 82: 831-836, 2012.

16 Mourad WF, Packianathan S, Shourbaji RA, Zhang Z, Graves M, Khan MA, Baird MC, Russell G and Vijayakumar S: The Impact of morbid (Class III) obesity on heterotopic ossification. Practical Rad Oncol 2: 1-6, 2012.

17 Mourad WF, Packianathan S, Shourbaji RA, Zhang Z, Graves M, Khan MA, Baird MC, Russell G and Vijayakumar S: A prolonged time interval between trauma and prophylactic radiation therapy significantly increases the risk of heterotopic ossification. Int J Radiation Oncol Biol Phys 82: 339-344, 2012.

18 Brooker AF, Bowerman JW, Robinson RA and Riley LH: Ectopic ossification following total hip replacement: Incidence and method of classification. J Bone Joint Surg Am 55: 16291632, 1973.

19 Bossche LV and Vanderstraeten G: Heterotopic ossification: A review. J Rehabil Med 37: 129-136, 2005.

20 Riedel B: Demonstration line durch ach Hagiges Umhergehen total destruirten kniegelenkes von einem patienten mit stichverletzing des ruckans. Verh Dtsch Gesellschaft Chirurg 12: 93, 1883.

21 Dejerine A and Ceillier A: Para-osteo-arthropathies des paraplegigues par lesionmedullarie; etude clinique et radiographique. Ann Med, 1918.

22 Khan FM: Physics of Radiation Therapy. 4th ed. Philadelphia, PA: Lippincott Williams \& Wilkins; 2009.

23 Mourad WF, Saad AA, Hussein MA, Packianathan S, Z. Zhang Z, Shourbaji RA, Caudell JJ, Jennelle RL and Baird MC: Postradiation therapy sarcoma. J Clin Oncol 28(15): e20523, 2010.

24 Mourad WF, Packianathan S, Baird MC, Shourbaji RA, Khan MA, Akhtar I, Cheng S, Caudell J, Jenelle R and Vijayakumar $\mathrm{S}$ : Radiation-induced malignancy after prophylactic radiation therapy in prevention of heterotopic ossification. Int J Radiat Oncol Biol Phys 78(3): sP610, 2010.
25 Mourad WF, Packianathan S, Shourbaji RA, Russell G, Khan MA and Vijayakumar S: Radiation-induced sarcoma following radiation prophylaxis of heterotopic ossification. Practical Radiation Oncol 2: 151-154, 2012.

26 Farris MK, Chowdhry VK, Lemke S, Kilpatirck M and Lacombe M: Osteosarcoma following single fraction radiation prophylaxis for heterotopic ossification. Radiation Oncol 7: 140, 2012.

27 Bieri S, Rouzaud M and Marilbell R. Seminoma of the testis: Is scrotal shielding necessary when radiotherapy is limited to the para-aortic nodes? Radiother Oncol 3: 349-353,1999.

28 Mourad WF, Packianathan S, Yang CC, Shourbaji RA, Zhang Z, Kanakamedala MR, Khan MA, Mobit P, Katsoulakis E, Nbahani T, Jenelle R, Russell G and Vijayakumar: Computerized tomography-based radiotherapy improves heterotopic ossification outcomes. BONE 57(1): 132-136, 2013.

29 Freund I, Zenzes Mt, Müller R, Pötter R, Knuth Ua And Nieschlag E: Testicular function in eight patients with seminoma after unilateral orchidectomy and radiotherapy. Int J Andro 10(2): 447-455, 1987.

30 Fraass BA, Kinsella TJ, Harrington FS AND Glatstien E: Peripheral dose to the testes: The design and clinical use of a practical and effective gonadal shield. Intl J Radiation Oncol Biol Physics 11(3): 609-615, 1985.

31 McGinley PH: Shielding techniques for radiation oncology facilities. Madison, Wis: Medical Physics Publishing, 2002.

32 Mourad WF, Ma JK, Yang CC, He R, Ryniak M, Shourbaji RA, Mobit PN, Santoro J, Nabhani T, Katsoulakis E, Kabarriti R, Blakaj A, Patel S, Yan W, Blakaj D, Khan MA, Packianathan S, Jennelle RL, Russell GV and Vijayakumar S: Higher energy megavoltage photon and split beam technique minimize the testicular dose during radiation prophylaxis of heterotopic ossification. Int J Radiat Oncol Biol Phys 90(3): s684, 2014.

33 Mourad WF, Shourbaji RA and Packianathan S. Testicular dose during radiation prophylaxis of heterotopic ossification. American Radium Society annual meeting 2011.

34 Mourad WF, Shourbaji RA, Baird MC, Dieck G, Edwards J, Mobit P, Yang CC, Hu KS and Harrison LB: Ovarian radiation dose during heterotopic ossification radiation prophylaxis. Int $\mathrm{J}$ of Radiat Oncol Biol Phys 81(2): s640, 2011. 